\title{
Pulsations in massive stars: effect of the atmosphere on the strange mode pulsations
}

\author{
Mélanie Godart ${ }^{1}$, Marc-Antoine Dupret ${ }^{1}$, Arlette Noels ${ }^{1}$, Conny \\ Aerts $^{2}$, Sergio Simón-Díaz ${ }^{3,4}$, Karolien Lefever ${ }^{5}$, Joachim Puls ${ }^{6}$, \\ Josefina Montalban ${ }^{1}$ and Paolo Ventura ${ }^{7}$ \\ ${ }^{1}$ Astrophysics, Geophysics, and Oceanography Department, University of Liége, Belgium \\ email: godart@astro.ulg.ac.be; \\ ${ }^{2}$ Instituut voor Sterrenkunde, KULeuven, Belgium; ${ }^{3}$ Instituto de Astrofísica de Canarias, \\ E-38200, La Laguna, Tenerife, Spain; ${ }^{4}$ Departamento de Astrofísica, Universidad de La \\ Laguna, E-38205 La Laguna, Tenerife, Spain; ${ }^{5}$ Belgisch Instituut voor Ruimte Aeronomie \\ (BIRA), Brussels, Belgium; ${ }^{6}$ Universitäts-Sternwarte, Scheinerstrasse 1, 81679 München, \\ Germany; ${ }^{7}$ INAF Osservatorio Astronomico di Roma, Monteporzio, Italy
}

\begin{abstract}
Recent space observations with CoRoT and ground-based spectroscopy have shown the presence of different types of pulsations in OB stars. These oscillations could be due to acoustic and gravity modes, solar-like oscillations or even other pulsations of large growth rates. We present a first attempt at interpreting the latter as strange modes.
\end{abstract}

Keywords. stars: atmospheres, stars: mass loss, stars: oscillations (including pulsations)

\section{Introduction: strange modes properties}

Massive stars are expected to present $\beta$ Cephei type modes (low order $\mathrm{p}$ and g-modes with periods of the order of hours) and Slowly Pulsating B star (SPB) type modes (high order g-modes with periods around 1 day). Moreover, theoretical models predict, for highly luminous stars $\left(L / M \sim 10^{4}\right)$, so called strange mode pulsations (e.g. Saio et al. 1998). In this preliminary study, some strange mode properties are presented together with the effect of the atmosphere model on these pulsations. Strange mode oscillations are trapped into a cavity caused by a density inversion located near the iron opacity bump. This inversion appears in the external region of high $L / M$ ratio stars, at an optical depth $\tau$ of about 5.3 in log, where the radiation pressure plays an important role. The confinement of the pulsation in a small propagation region alters the behaviour of these modes compared to acoustic or gravity modes: the growth rate is very large and the dimensionless frequencies decrease along the evolution on the main sequence (MS), i.e. with a decreasing effective temperature $T_{\mathrm{e}}$ (Fig. 1). As the MS evolution proceeds, the cavity widens and the trapping becomes less effective (Godart et al., 2010).

\section{Effect of the atmosphere: FASTWIND v.s. Eddington}

When considering a dynamic atmosphere model, FASTWIND (Puls et al. 2005), rather than a static grey Eddington atmosphere, a temperature $(T)$ inversion appears at $\log \tau \sim$ -2.0 and, therefore, a new trapping region occurs. The $T$ inversion is more pronounced when located at smaller $\tau$, hence the "atmospheric" trapping cavity is more efficient. To characterize the wind, we use the wind strength parameter, $\log Q=\log \left[\dot{M} /\left(R v_{\infty}\right)^{1.5}\right]$, whose value is $\log Q \simeq-15(\log Q \simeq-12)$ for a negligible (resp. strong) wind. Fig. 2 illustrates the cavity dimension in function of $\log Q$ and $\log T_{\mathrm{e}}$; the point size is proportional to its dimension expressed in $\tau$. The location and extent in $\tau$ of the $T$ inversion is 


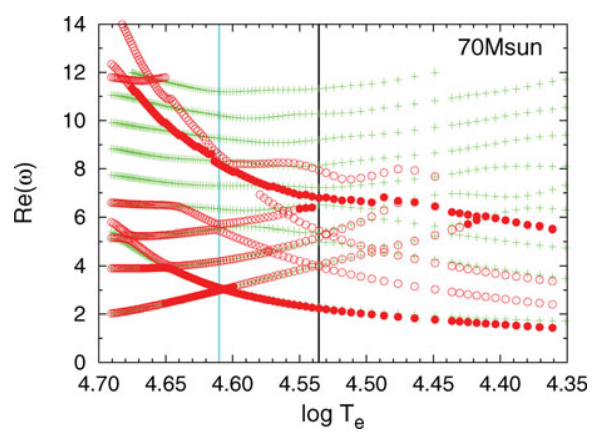

Figure 1. Adiabatic and non-adiabatic frequency spectrum (crosses and circles respectively) computed with the MAD oscillation code (Dupret et al. 2002) for a $70 \mathrm{M}_{\odot}$ star computed with the ATON evolutionary code (Ventura et al. 2008). Full circles stand for excited modes.

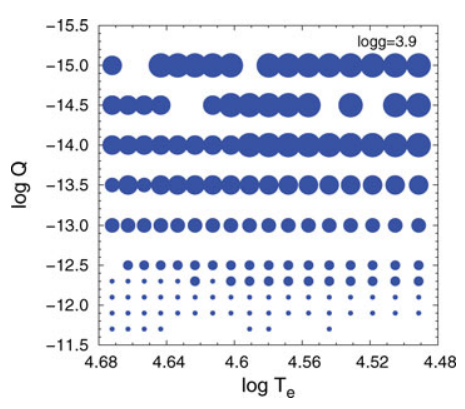

Figure 2. Effect of the wind strength parameter (Q) on the temperature inversion location. The point size is proportional to the cavity dimension expressed in optical depth. The smallest dots stand for a cavity located at $\log \tau>-1$, and the largest ones stand for $\log \tau<-2.5$.

mostly sensitive to $\log Q$. The cavity is thus more effective for weaker winds. We check the effect of the atmosphere model for $40 \mathrm{M}_{\odot}$ and $70 \mathrm{M}_{\odot}$ at the beginning (ZAMS), the middle (MMS) and the end of the MS (TAMS). No effect from the atmosphere model is noticeable on the strange mode eigenfunctions and frequencies for the $40 \mathrm{M}_{\odot}$ models. For the $70 \mathrm{M}_{\odot}$ star, no strange mode is found at the ZAMS and the spectrum is not altered by the different atmospheres. Excited strange modes are found in the $70 \mathrm{M}_{\odot} \mathrm{MMS}$ model, independently of the atmosphere model and the frequencies and eigenfunctions are similar. The "iron bump" cavity of the strange mode propagation then widens and in the $70 \mathrm{M}_{\odot}$ TAMS model, the associated strange modes have lost their strange mode behaviour. However, thanks to the new "atmospheric" cavity, an excited strange mode is found, whereas it is obviously not present in the model with an Eddington atmosphere.

\section{Conclusion}

During most of the MS phase, for stars from 40 to $70 \mathrm{M}_{\odot}$, we find excited strange modes, indenpendently of the considered atmosphere model, due to the presence of a trapping cavity near the iron opacity bump. These models are located in a HR diagram region corresponding to $\mathrm{O}$ stars, which have been observed by the CoRoT space mission. However no such modes have yet been detected. On the contrary, from CoRoT photometry and from spectroscopy, Aerts et al. (2010) detected a strange mode candidate in the B6Ia HD 50064: our $70 \mathrm{M}_{\odot}$ TAMS model is located in the same region of the HR diagram. However this model can only excite strange mode with an "atmospheric" cavity, i.e. with a FASTWIND atmosphere model. In that case, we find an excited strange mode with a period of 15 days (for indication only). Such a strange mode could be susceptible of inducing mass loss.

\section{References}

Aerts, C., Lefever, K., Baglin, A., \& Degroote, P. et al. 2010, A\&AA, 513, L11

Dupret, M.-A., De Ridder, J., Neuforge, C., \& Aerts, C. et al. 2002, A\&A, 385, 563

Godart, M., Dupret, M.-A., \& Noels, A., et al. 2010, AN, in press

Puls, J., Urbaneja, M. A., Venero, R., Repolust, T. et al. 2005, A\&AA, 435, 669

Ventura, P., D'Antona, F., \& Mazzitelli, I. 2008, ApESSS, 316, 93

Saio, H., Baker, N. H., \& Gautschy, A. 1998, MNRAS, 294, 622 